\title{
Helden oder Staatsverräter? Zur sprachlichen Konstruktion vom Bild der ukrainischen Maidan-Aktivisten im deutschen, polnischen und russischen Mediendiskurs - eine kognitive Studie
}

\author{
Agnieszka Gawel (Kraków)
}

\begin{abstract}
Cognitive linguistics pays particular attention to the interrelations between language, subjective conceptualisations and intersubjective manifestations of knowledge, providing descriptive tools for the analysis of how the choice of linguistic means is determined by individual, as well as by culture-specific conceptual patterns. In this paper we analyse the linguistic construal of Ukrainian Maidan activists in six German, Polish and Russian daily newspapers. The analysis focuses on culture-specific differences between the linguistic strategies used in the Western and Middle European media discourse in the EU member states, as well as the Eastern European discourse in Russia. The research is based on two theoretical frameworks: cognitive ethnolinguistics and cognitive grammar.
\end{abstract}

\section{$1 \quad$ Einleitung}

Die im Rahmen der kognitiven Linguistik vorgenommene Forschung zu Wechselbeziehungen zwischen Sprache, subjektiven Konzeptualisierungen und intersubjektiven Denkmustern liefert ein geeignetes Werkzeug für die Analyse von sprachlichen Reflexen kulturspezifischer Sichtweisen, wie sie im Mediendiskurs zum Ausdruck kommen. Im vorliegenden Text unternehmen wir den Versuch, das Bild der Maidan-Aktivisten im deutschen, polnischen und russischen Diskurs innerhalb von sechs Tageszeitungen zu analysieren. Der Schwerpunkt liegt dabei auf der Identifikation von kulturspezifischen Unterschieden zwischen der sprachlichen Konstruktion vom Bild der Demonstranten, die für den west- sowie mitteleuropäischen Diskurs in den EUMitgliedstaaten und den osteuropäischen Diskurs in Russland charakteristisch ist. Die theoretische Grundlage für die vorliegende Analyse bilden zwei kognitive Ansätze: die kognitive Ethnolinguistik und die Kognitive Grammatik.

\section{$2 \quad$ Zur Relevanz der Kognitiven Linguistik für die Diskursanalyse}

Die holistischen Ansätze in der Kognitiven Linguistik stellen die Wechselbeziehungen zwischen Sprache und Konzeptualisierung in den Mittelpunkt sprachwissenschaftlicher Forschung. Eine solche Formulierung des Untersuchungsgegenstands erlaubt die These, dass Bedeutungen sprachlicher Ausdrücke als Konzepte zu betrachten sind, welche die mentale Erfahrung der 
Sprachnutzer wiederspiegeln. In zahlreichen Positionen zur Anwendbarkeit der kognitiven Linguistik in der Diskursforschung wird auf den zweiseitigen Charakter der Konzeptualisierungen hingewiesen, die sich in der Sprache manifestieren. Die anhand sprachlicher Daten rekonstruierbaren Denkmuster und Wissenssegmente haben einerseits subjektiven Charakter, da sie in der Kognition der einzelnen Individuen repräsentiert sind. Andererseits werden sie infolge der Vergesellschaftung zum konzeptuellen Erbgut der gesamten Sprach- und Kulturgemeinschaft (cf. z. B. Fraas/Meier 2004; Fraas 2005: 243; Busse 2008: 78).

Die Platzierung der Wechselbeziehungen zwischen Sprache, Kognition und Kultur in den Mittelpunkt sprachwissenschaftlicher Forschung zieht die Frage nach sich, welche Methode dem Linguisten einen optimalen Zugriff auf die sich im Diskurs manifestierenden und anhand von Sprache rekonstruierbaren kulturspezifischen Denkmuster erlaubt. Da eine Besprechung sämtlicher Methoden zur Modellierung sprachlicher Reflexe kulturspezifischer Sichtweisen, die im Rahmen der Kognitiven Linguistik bearbeitet wurden, den Rahmen des vorliegenden Beitrags sprengen würde, beschränken wir uns hier auf zwei kognitive Ansätze, welche die Grundlage für die von uns vorgenommene Analyse darstellen: die Kognitive Grammatik und die kognitive Ethnolinguistik.

\subsection{Die Kognitive Grammatik in der Analyse des Mediendiskurses}

Auf die Relevanz von Erkenntnissen der Kognitiven Grammatik für die Diskursforschung verweist Langacker (2001; 2008), der die für Untersuchungen zur Diskursanalyse zentrale These formuliert, dass alle sprachlichen Einheiten aus Gebrauchsereignissen abstrahiert würden und aus diesem Grund immer in einem Diskurskontext eingebettet seien. Der Diskurs bestehe aus mehreren Gebrauchsereignissen, die als „Fälle des Gebrauchs von Sprache in ihrer gesamten Komplexität und Spezifität"1 definiert werden können (Langacker 2008: 457). Von zentralem Interesse für die kognitiven Untersuchungen zum Mediendiskurs ist die Frage, inwieweit und auf welche Weise die semantische Charakteristik von Gebrauchsereignissen die subjektiven und intersubjektiven Aspekte der Konzeptualisierung wiederspiegelt. Die Subjektivität der Konzeptualisierungen ergibt sich daraus, dass die Kommunikationspartner ihre Aufmerksamkeit ausschließlich auf bestimmte Aspekte der objektiv stattgefundenen Situation richten. Diese Einengung der Betrachtungsperspektive, die aufgrund des selektiven Charakters der Aufmerksamkeit unvermeidbar ist, wird bei Langacker (2001: 145) metaphorisch mit der Wahrnehmung der Wirklichkeit durch ein Fenster mit einem bestimmten, im Voraus festgesetzten Rahmen verglichen: ,[...] es ist, als würden wir die Welt durch ein Fenster betrachten oder, anders gesagt, durch einen Betrachtungsrahmen“". ${ }^{2}$

Damit ein erfolgreicher Kommunikationsakt zustande kommt, müssen jedoch die in der Kognition der Individuen entstandenen Konzeptualisierungen bestimmte Gemeinsamkeiten aufweisen. Daher setzt der Diskurs immer die Intersubjektivität gewisser Wissensbestände und Denk-

\footnotetext{
${ }^{1}$ Im Original: „,instances of language use in all their complexity and specificity“ (Langacker 2008: 457; Übers. d. Verf.).

${ }^{2}$ Im Original: ,[...] it is as if we are 'looking at' the world through a window, or 'viewing frame'." (Langacker 2001: 145; Übers. d. Verf.).
} 
muster voraus. Die Gesamtheit von Aspekten der Konzeptualisierung, die den Diskursteilnehmern gemeinsam sind und die Grundlage für die Kommunikation darstellen, bildet den aktuellen Diskursraum, d. h. den mentalen Raum, in dem der jeweilige Diskurs stattfindet (cf. Langacker 2001: 144f.; 2008: 466).

Der Terminus Diskurs wird bei Langacker grundsätzlich in zwei Lesarten verwendet: zur Bezeichnung einer Konversation (im Sinne der Gesprächsanalyse, cf. Langacker 2001) bzw. in Bezug auf die Ebene der Sprachbeschreibung, auf der ,eine beliebige Menge von Sätzen (bzw. ihren Fragmenten) in ein kohärentes sprachliches Gebilde in Form einer Konversation, eines Monologs [...] bzw. eines Schrifttextes umgestaltet werden kann“33 (Langacker 2008: 457). Das im Rahmen der Kognitiven Grammatik entwickelte Diskursmodell ist jedoch auch für Untersuchungen anwendbar, denen ein weiterer Diskursbegriff zugrunde liegt, der auch das in der deutschsprachigen Fachliteratur geprägte Verständnis des Diskurses als einer Textsammlung (cf. Warnke 2002: 131; Heinemann/Heinemann 2002) bzw. als eines vom Linguisten selegierten Korpus (cf. Busse/Teubert 1994: 14; Spieß 2011: 110f.) umfasst (cf. Pędzisz 2012, 2014; Gaweł 2016). Was die Untersuchungen zum Mediendiskurs anbelangt, verdient das Konzept des aktuellen Diskursraumes besonderer Beachtung, da es auf die intersubjektiven Aspekte der den Gebrauchsereignissen zugrunde liegenden Konzeptualisierungen Bezug nimmt. Die Annahme, dass der Diskurs in einem mentalen Raum stattfindet, der den Teilnehmern gemeinsam ist, erlaubt nämlich die These, dass die Kognitive Grammatik einen wesentlichen Beitrag zur Beschreibung der kollektiven Wissensbestände und Denkmuster leisten kann, welche verschiedene, für die einzelnen Diskursgemeinschaften charakteristische mentale Räume konstituieren.

\subsection{Die kognitive Ethnolinguistik in der Analyse des Mediendiskurses}

Der zweite linguistische Ansatz, der die Grundlage für die hier vorgenommene Analyse bildet, ist die kognitive Ethnolinguistik, insbesondere das von Jerzy Bartmiński (cf. z. B. Bartmiński 2006; 2012) entwickelte kognitiv orientierte Konzept des sprachlichen Weltbildes. Mit dem Terminus sprachliches Weltbild ${ }^{4}$ bezeichnet Bartmiński (2012: 266) eine ,in der Sprache enthaltene Wirklichkeitsinterpretation, die sich als Menge von Denkmustern über die Welt, Menschen, Gegenstände und Ereignisse erfassen lässt“. Diese Wirklichkeitsinterpretation zeichnet sich durch drei Eigenschaften aus: die Subjektivität, die Intersubjektivität und die Fähigkeit, sich in der Sprache zu manifestieren (cf. Bartmiński 2012: 266).

Die erstgenannte Eigenschaft, die Subjektivität, bezieht sich auf die Tatsache, dass das sprachliche Weltbild in der Kognition der Individuen verankert ist. Aus diesem Grund determiniert es das Sprachverhalten der einzelnen Mitglieder der jeweiligen Sprach- und Kulturgemeinschaft.

\footnotetext{
${ }^{3}$ Im Original: „The next level is discourse, where any number of sentences (or fragments thereof) are connected to form a coherent linguistic production - be it a conversation, a monolog [...], or a written text." (Langacker 2008: 457; Übers. d. Verf.).

${ }^{4}$ Im vorliegenden Beitrag stützen wir uns auf das im Rahmen der kognitiven Ethnolinguistik entwickelte Konzept des sprachlichen Weltbildes. Für detaillierte Informationen zu Ursprüngen der Forschung zum sprachlichen Weltbild cf. Humboldt (1836), Weisgerber (1950), Whorf (1956).
} 
Die Intersubjektivität der in der Sprache enthaltenen Wirklichkeitsinterpretation ist eng mit der Tatsache verbunden, dass bestimmte Denkmuster der Vergesellschaftung unterliegen. Die Mitglieder der jeweiligen Sprach- und Kulturgemeinschaft tendieren folglich dazu, sprachliche Ausdrucksmittel auszuwählen, welche die für ihre Kultur charakteristischen Denkmuster ausdrücken. Grundsätzlich können im Hinblick auf die Art der Vergesellschaftung drei Typen des sprachlichen Weltbildes unterschieden werden: das für eine Nation bzw. eine übernationale Sprachgemeinschaft charakteristische, das gruppenspezifische und das zeitspezifische Weltbild. ${ }^{5}$

Die drittgenannte Eigenschaft, die Fähigkeit, sich in der Sprache zu manifestieren, bezieht sich auf die Tatsache, dass die erwähnte Wirklichkeitsinterpretation in der Sprache enthalten und anhand von sprachlichen Daten rekonstruierbar ist.

Im Hinblick auf den Untersuchungsgegenstand des vorliegenden Beitrags verdient auch das Konzept des diskursiven Weltbildes besonderer Beachtung. Der Terminus diskursives Weltbild dient zur Bezeichnung einer anhand der Diskursanalyse rekonstruierbaren Wirklichkeitsinterpretation, die einerseits im Sprachsystem verankert ist, andererseits aber diskursiv erzeugt wird (cf. Czachur 2011: 144f., 147; 2013: 188). Das diskursive Weltbild spiegelt immer eine bestimmte, für die jeweilige Diskursgemeinschaft charakteristische, kulturspezifische Sichtweise wider.

\section{Gesellschaftlich-politischer Hintergrund}

Den Anstoß für die im vorliegenden Beitrag vorgenommenen Untersuchungen bildeten die Beobachtungen der Textverfasserin zur Medienberichterstattung über die sog. Euromaidan-Bewegung in der deutschen, polnischen und russischen Tagespresse. Als Euromaidan wird, insbesondere im mittel- und westeuropäischen Diskurs, die zwischen November 2013 und Februar 2014 stattgefundene Protestbewegung der ukrainischen Bevölkerung gegen die prorussische Politik der Regierung unter dem damaligen Präsidenten Viktor Janukowitsch bezeichnet (nach der Selbstbezeichnung der Aktivisten ,Revolution der Würde' (ukr. Революиія гідності)). Zum Ausbruch der Proteste kam es in der Nacht vom 21. auf den 22. November 2013 nach der überraschenden Ablehnung der Unterzeichnung des Assoziierungsabkommens mit der Europäischen Union seitens der ukrainischen Regierung und der Ankündigung von Gesprächen über ein Assoziierungsabkommen mit Russland. Die Demonstrationen gewannen immer mehr an Reichweite und endeten am 22. Februar mit der Amtsenthebung des damaligen Präsidenten der Ukraine Viktor Janukowitsch und der Festlegung des Termins vorgezogener Neuwahlen.

Die Proteste stießen auf große Resonanz in den europäischen Medien, wobei zwischen der medialen Berichterstattung in den EU-Mitgliedstaaten und Russland wesentliche Unterschiede aufgetreten sind, die sowohl die Auswahl der dargestellten Ereignisse, wie auch die Sprachgestaltung des Diskurses betrafen. Der Zweck des vorliegenden Beitrags besteht in der Analyse dieser Diskrepanzen, wobei der Mediendiskurs über die Euromaidan-Bewegung exemplarisch

\footnotetext{
${ }^{5}$ Für nähere Informationen zu den zwei erstgenannten Typen des sprachlichen Weltbildes, die sich aus dem zwischensprachlichen und innersprachlichen Relativismus ergeben, cf. Tokarski/Nowak (1999: 209f.). Auf das Vorhandensein des zeitspezifischen sprachlichen Weltbildes verweist Gaweł (2020: 4) in ihren Untersuchungen zur sprachlichen Konstruktion des Brexit-Diskurses.
} 
als ein besonders deutlicher Fall der bewussten Konstruktion von diskursiv zentralen Gegenständen betrachtet wird. Das Hauptaugenmerk der Textverfasserin liegt folglich nicht auf der Bewertung der Berichterstattung im mittel- sowie westeuropäischen Diskurs in den EU-Mitgliedstaaten und im osteuropäischen Diskurs in Russland, sondern auf der Illustrierung sprachlicher Mechanismen der Wirklichkeitskonstruktion. Im Fokus der Überlegungen steht dabei ein Leitgedanke Foucaults:

Ich setze voraus, daß in jeder Gesellschaft die Produktion des Diskurses zugleich kontrolliert, selektiert, organisiert und kanalisiert wird - und zwar durch gewisse Prozeduren, deren Aufgabe es ist, die Kräfte und die Gefahren des Diskurses zu bändigen, sein unberechenbar Ereignishaftes zu bannen, seine schwere und bedrohliche Materialität zu umgehen.

(Foucault 1991: 10f.).

\section{Das Bild der Demonstranten im deutschen, polnischen und russischen Mediendis- kurs zum Konflikt in der Ukraine - eine empirische Analyse}

Im vorliegenden Teil des Artikels konzentrieren wir uns auf die Rekonstruktion vom Bild der Demonstranten im deutschen, polnischen und russischen Mediendiskurs zum Konflikt in der Ukraine. Das Korpus für die Analyse bildet eine Sammlung von Presseartikeln, die zwischen dem 21. November 2013 (Ablehnung der Unterzeichnung vom Vertrag mit der Europäischen Union seitens der ukrainischen Regierung) und dem 22. Februar 2014 (Verlassen der Residenz in Kiew durch den ukrainischen Präsidenten Viktor Janukowitsch) in den Internetportalen der folgenden Tageszeitungen erschienen sind: Dziennik Polski, Gazeta Wyborcza, die Frankfurter Allgemeine Zeitung, die Süddeutsche Zeitung, Российская Газета und Известия. ${ }^{6}$ Im Mittelpunkt unserer Überlegungen stehen folgende Fragen:

- Welche Aspekte vom diskursiven Bild der Demonstranten können im analysierten Korpus identifiziert werden?

- Welche sprachlichen Ausdrucksmittel werden bei der Konstruktion der einzelnen Aspekte vom Bild der Demonstranten verwendet?

- Welche kulturspezifischen Unterschiede zwischen der für den deutschen, polnischen und russischen Mediendiskurs charakteristischen sprachlichen Konstruktion vom Bild der Demonstranten sind im analysierten Material zu beobachten?

\subsection{Demonstranten als Subjekte, welche die Realisierung ihrer politischen Postulate anstreben}

Der erste Aspekt des diskursiven Bildes von den ukrainischen Aktivisten, der in der polnischen und deutschen Presse im Vordergrund steht, ist jener von Subjekten, welche die Realisierung ihrer politischen Postulate anstreben. Er kommt oft bereits in Bezeichnungen für die Protestierenden zum Ausdruck. Die Demonstranten werden typischerweise entweder als Oppositionsbewegung gegen die ukrainische Regierung konzeptualisiert: z. B. „uczestnicy protestów antyrządowych“" [,Teilnehmer an Protesten gegen die Regierung‘] (Bielecki/Tymots 2014, GW),

\footnotetext{
${ }^{6}$ Im weiteren Text des Beitrags verwenden wir beim Zitieren der genannten Zeitungen die folgenden Abkürzungen: SZ - Süddeutsche Zeitung, FAZ - Frankfurter Allgemeine Zeitung, GW - Gazeta Wyborcza, DP - Dziennik Polski, РГ - Российская Газета.
} 
„Regimegegner“ ( $\mathrm{NN}_{1}$ 2014, SZ) oder als Befürworter der engen Zusammenarbeit mit der Europäischen Union: „Zwolennicy integracji europejskiej“ [,Befürworter der europäischen Integration“] (Junko 2014a, DP), „die proeuropäische Oppositionsbewegung“ (Schuller 2014, FAZ).

$\mathrm{Zu}$ den sprachlichen Ausdrucksmitteln, die bei der Konstruktion des genannten Subbildes von Protestteilnehmern verwendet werden, gehören auch Bezüge auf Aussagen ukrainischer Demonstranten und prominenter Unterstützer der ukrainischen Oppositionsbewegung. Ein charakteristisches Merkmal dieser Äußerungen ist die Verwendung von Verben in der 1. und 2. Person Plural, was eine enge Beziehung zwischen dem Sender der Äußerung, den Demonstranten und - im Falle der 1. Person Plural - der Gesamtheit von ukrainischen Bürgern signalisiert:

Außer Euch hat die Ukraine keine Verteidiger. Kämpft! Ihr seid Helden.

$\left(\mathrm{NN}_{4} 2014, \mathrm{SZ}\right)$

Powinniśmy szybko nawiazać takie relacje z UE, jakie ma teraz Norwegia.

[,Wir sollten rasch solche Beziehungen mit der EU anknüpfen, wie Norwegen sie gegenwärtig hat.']

(Wieliński 2014, GW)

Darüber hinaus wird die pro-europäische Haltung der Demonstranten in vielen Textauszügen thematisiert, in denen Hintergrundinformationen zu dargestellten Ereignissen explizit angeführt werden:

Die Demonstranten kritisieren die Entscheidung von Präsident Viktor Janukowitsch, ein über Jahre ausgehandeltes Assoziierungsabkommen mit der EU nicht zu unterzeichnen.

$\left(\mathrm{NN}_{3}, \mathrm{FAZ}\right)$

Protestuja oni już niemal trzy miesiące przeciw decyzji wtadz, które nagle wycofaly się $z$ podpisania umowy stowarzyszeniowej z Uniq Europejska i postanowily zacieśnić relacje z Rosja. [, Sie protestieren schon beinahe drei Monate gegen die Entscheidung der ukrainischen Staatsgewalt, die plötzlich ihre Zusage zum Assoziierungsabkommen mit der Europäischen Union zurückzog und beschloss, engere Beziehungen mit Russland einzugehen.']

(Kublik 2014, GW)

In den analysierten Artikeln aus russischen Tageszeitungen ist das Subbild der Demonstranten als Kämpfer für die ukrainische Souveränität bzw. pro-europäische Bürgerbewegung entweder überhaupt nicht vertreten oder es wird marginalisiert. Dies wird bereits aus der Analyse der im russischen Subkorpus auftretenden Bezeichnungen für die ukrainischen Aktivisten ersichtlich, die lediglich die Aktivität des Protestierens bzw. die Anteilnahme am politischen Geschehen profilieren7, ohne auf den Zweck der Proteste zu verweisen (z. В. „демонстранты“ “, Demonstranten“] (Лубенский/Соколовская 2013, Известия), „участники акций протеста“ [,Teilnehmer an der Protestaktion“] (Дульман/Макарычев 2014, РГ), „митингующие“ [, Demonstranten“] (Дульман 2014b, РГ), „протестующие“ [,Protestierende“] (Соколовская 2014, Известия), ,активисты майдана““, [Aktivisten des Majdan“] (NN6 2014, Известия).

\footnotetext{
${ }^{7}$ Den Terminus ,,profilieren“ verwenden wir hier - entsprechend seinem Gebrauch in der Kognitiven Grammatik - in Bezug auf die Hervorhebung einer semantischen Substruktur (des Profils) innerhalb einer größeren semantischen Struktur (der Basis; cf. Langacker 2008: 66).
} 
Auch Bezüge auf Aussagen ukrainischer Aktivisten und anderer prominenter Gestalten, welche die ukrainische Protestbewegung unterstützen, kommen im genannten Subkorpus nur vereinzelt vor. In diesen selten anzutreffenden Verweisen wird - im Unterschied zur deutschen und polnischen Tagespresse - die Verwendung der 1. bzw. 2. Person Plural zur Hervorhebung der Solidarität mit den Demonstranten bzw. der Gesamtheit ukrainischer Bürger grundsätzlich vermieden:

Как только я узнал о заявлении премьера Азарова не подписывать согламение с Евросоюзом, моим первым желанием было взяться за вильы [...].

[,Als ich von der Erklärung von Ministerpräsident Asarow über die Nichtunterzeichnung des Abkommens mit der Europäischen Union erfahren habe, wollte ich sofort zur Forke greifen [...].']

(Макарычев 2013, РГ)

Explizite Kommentare, die Hintergrundinformationen zu Zielen und Ursachen der Aktivisten enthalten, sind in Presseartikeln, die auf die Anfangsphase der Proteste Bezug nehmen, häufig anzutreffen. Textpassagen, Sätzen und Satzteilen, in denen Motive der Demonstranten geschildert werden, folgen jedoch typischerweise Textstellen, in denen die Schwächen der ukrainischen Protestbewegung im Vordergrund stehen:

Владислав Вдовин - из Львова, где хотят «новую Украину», но не знают, как ее строить [,Vladislav Vdovin - aus Lemberg, wo sie eine neue Ukraine wollen, aber nicht wissen, wie man sie aufbauen soll']

(Вдовин 2013, Известия)

Da bei der Darstellung der weiteren Phasen der Maidan-Bewegung in der russischen Tagespresse die Radikalisierung der ukrainischen Aktivisten in den Vordergrund gestellt wird, werden in den späteren Artikeln zu Demonstrationen in der Ukraine explizite Hintergrundinformationen zu politischen Postulaten der Aktivisten immer seltener angegeben.

\subsection{Demonstranten als Vertreter der Gesellschaft}

Ein weiterer Aspekt des diskursiven Bildes von den ukrainischen Aktivisten, der für das deutsche und polnische Subkorpus charakteristisch ist, sind Demonstranten als Vertreter der Gesellschaft. $\mathrm{Zu}$ seinen sprachlichen Exponenten gehören metonymische Verweise auf die Protestierenden, welche die kognitiven Domänen der Menschen und Ukrainer aktivieren:

Zehntausende Menschen waren einem Protestaufruf der inhaftierten Oppositionspolitikerin Julia Timoschenko gefolgt.

$\left(\mathrm{NN}_{5} 2013, \mathrm{SZ}\right)$

Ukraińców nie da się złamać

[,Die Ukrainer lassen sich nicht brechen']

(Wieliński 2014, GW)

Das analysierte Korpus enthält Bezüge auf die hohe Anzahl von Demonstranten, was impliziert, dass die ukrainischen Proteste von breiten Bevölkerungskreisen unterstützt werden:

Trotz Demonstrationsverbots harrten abermals tausende Regierungsgegner in der Nacht bei eisigen Temperaturen im Stadtzentrum aus.

$\left(\mathrm{NN}_{3} 2014, \mathrm{FAZ}\right)$ 
Protestuje kilkaset tysięcy osób

[,Einige Hunderttausend Menschen protestieren']

(Kołodyński 2013, DP)

Das Subbild der Protestierenden als Vertreter der Gesellschaft konstituieren auch Ausdrücke, die auf die Repräsentativität der Demonstranten für die gesamte ukrainische Bevölkerung Bezug nehmen:

Sie kamen von überallher, um die Stadt und ihre Ukraine gegen die Regierung und ihr diktatorisches Verhalten gegenüber dem eigenen Volk zu verteidigen.

(Petrowskaja 2014, FAZ)

Demonstranci reprezentuja cały przekrój ukraińskiego społeczeństwa. Sa rosyjsko- $i$ ukraińskojęzyczni, z miast i ze wsi, ze wszystkich zakątków kraju i orientacji politycznych, młodzi i starzy, chrześcijanie, muzutmanie, Żydzi.

[,Die Demonstranten vertreten den gesamten ukrainischen Bevölkerungsquerschnitt. Es gibt Russisch- und Ukrainischsprachige, Stadt- und Landbewohner. Sie kommen aus allen Ecken des Landes, sie vertreten sämtliche politischen Orientierungen, es gibt Junge und Alte, Christen, Moslems und Juden.']

(Snyder 2014, GW)

Darüber hinaus sind im deutschen und polnischen Korpus zahlreiche Belege zu finden, in denen das Ausmaß der Proteste profiliert wird, ohne explizit auf die Anzahl bzw. Repräsentativität der Teilnehmer zu verweisen:

Denn der Aufstand gegen die Regierung hatte am Samstag schon das halbe Land ergriffen.

(Kahlweit 2014a, SZ)

Gdy popatrzeć na mape Ukrainy z przejętymi obwodami, można odnieść wrażenie, że wrze pót kraju.

[,Wenn man auf die ukrainische Landkarte mit übernommenen Bezirken schaut, bekommt man den Eindruck, dass es in der Hälfte des Landes gärt.']

(Bielecki/Tymots 2014, GW)

Im russischen Subkorpus ist das Subbild von Demonstranten als Vertreter der Gesellschaft grundsätzlich nicht vorhanden. In den analysierten Artikeln aus Российская Газета und Известия beobachten wir einen vollständigen Verzicht auf die metonymische Verwendung der Bezeichnungen Menschen und Ukrainer in Bezug auf die Protestierenden. Auch Belege, in denen das Ausmaß der Proteste thematisiert wird, treten nur vereinzelt auf. Stattdessen sind im russischen Subkorpus zahlreiche Textstellen zu finden, in denen die geringe Anzahl und fehlende Repräsentativität der Maidan-Aktivisten profiliert werden:

Украинский народ на майдане есть. Но лишь небольшая часть украинского народа.

[,Die ukrainische Nation ist auf dem Maidan. Allerdings nur ein geringer Teil der ukrainischen Nation.']

(Лимонов 2014, Известия)

Auch wenn in vereinzelten Belegen Bezüge auf das breite Ausmaß der Proteste auftreten, werden sie in der Regel von sprachlichen Ausdrucksmitteln begleitet, mit deren Hilfe ihre Rechtswidrigkeit bzw. Gewalttätigkeit hervorgehoben werden: 
На Украине десятки тысяч людей вновь вышли на площчадь Независимости, протестуя против отказа властей подписать договор о сотрудничестве с Евросоюзом, передает РИА Новости. [...] Между тем участники акиии разбили несколько окон в здании Киевской городской администраџии [...].

[,In der Ukraine kamen Zehntausende von Menschen auf den Unabhängigkeitsplatz, um gegen die Ablehnung der Unterzeichnung des Assoziierungsabkommens mit der EU durch die Regierenden zu protestieren - so die Presseagentur Novosti. [...] Inzwischen haben die Teilnehmer an der Protestaktion einige Fenster im Kiewer Verwaltungsgebäude zerschlagen [...].']

$\left(\mathrm{NN}_{8} 2013, \mathrm{P \Gamma}\right)$

\subsection{Demonstranten als gefährliche und gewaltbereite Rebellen}

Ein weiterer Aspekt des diskursiven Bildes von den ukrainischen Aktivisten ist jener von Demonstranten als gefährlichen, gewalttätigen Rebellen. Bezüge auf die Gewaltbereitschaft der Protestierenden treten in allen analysierten Zeitungen auf. Sie unterscheiden sich jedoch hinsichtlich der Art und Weise der Darstellung von diesem Aspekt der konstruierten Szene in der jeweiligen Quelle. In den polnischen und deutschen Zeitungen werden sie typischerweise von sprachlichen Ausdrucksmitteln begleitet, die der Marginalisierung des genannten Subbildes der ukrainischen Aktivisten dienen. Zu diesen gehören:

- Sequentielle Ikonizität

In den meisten Sätzen, in denen die Gewaltbereitschaft von beiden Seiten des Konflikts thematisiert wird, stehen Bezüge auf die seitens der Sicherheitskräfte ergriffenen Maßnahmen vor den Verweisen auf die Demonstranten:

Snajperzy strzelają do tłumu. Demonstranci również atakują.

[,Die Scharfschützen schießen in die Menge. Auch die Demonstranten greifen an.']

(Knap 2014, DP)

Immer wieder werfen Polizisten Blendgranaten und offenbar auch Molotowcocktails in die Menge, die wirft zurück.

(Kahlweit 2014b, SZ)

- Sprachliche Ausdrucksmittel, welche die Auslassung des Agens ermöglichen und dadurch die Anonymisierung der Aussage gewährleisten:

U wejścia do budynku administracji doszło do brutalnej bójki z milicjantami z oddziałów Gryfon, którzy nie wpuszczali protestujących do środka [...].

[,Am Eingang zum Verwaltungsgebäude kam es zu einer brutalen Schlacht mit den Polizisten von der Sondereinheit „Griffon“, die die Protestierenden nicht einließen [...].']

(Junko 2014b, DP)

In der Stadt Tscherkassy kam es zu schweren Zusammenstößen zwischen Demonstranten und Polizei.

$\left(\mathrm{NN}_{2} 2014, \mathrm{FAZ}\right)$ 
- Ausdrücke, die explizit auf das seltene Auftreten von Gewalttaten seitens der Demonstranten verweisen:

Vereinzelt schleuderten Demonstranten Steine und Brandsätze auf Polizisten.

$\left(\mathrm{NN}_{3} 2014, \mathrm{FAZ}\right)$

Demonstranci od czasu do czasu strzelali w jego strone fajerwerkami, palili opony, ale nie atakowali funkcjonariuszy

[,Die Demonstranten schossen ab und zu Feuerwerkskörper in dessen Richtung [des Berkut, die Verf.], sie verbrannten Autoreifen, sie griffen jedoch die Polizeibeamten nicht an.']

(Radziwinowicz 2014, GW)

In den analysierten Artikeln aus der russischen Presse treten keine sprachlichen Ausdrucksmittel auf, welche die Marginalität von Gewalttaten der Demonstranten implizieren würden. In Bezügen auf die Brutalität der ukrainischen Aktivisten werden - mit ähnlicher Häufigkeit zwei Typen von Konstruktionen verwendet:

- Sätze ohne Agens, in denen die für die Gewalttaten zuständigen Täter unerwähnt bleiben:

В ходе массовых беспорядков в центре Киева пятеро военнослужащих внутренних войск были убиты из огнестрельного оружия.

[, Während der Massenunruhen im Zentrum von Kiew wurden fünf Soldaten der internen Truppen von Schusswaffen getötet.']

(Дульман/Макарычев 2014, РГ)

- Aktivische Sätze, in denen die für die Gewalt verantwortlichen Täter profiliert werden:

Протестующие используют боевое оружие.

[,Die Protestierenden setzen militärische Bewaffnung ein. ']

(Соколовская 2014, Известия)

Es sei hier jedoch betont, dass die sprachlichen Konstruktionen, mit deren Hilfe die Aussage anonymisiert wird, im russischen Subkorpus eine andere Funktion erfüllen als in der deutschen und polnischen Tagespresse. In den deutsch- und polnischsprachigen Artikeln dienen sie der Platzierung von den Gewalttätern im Hintergrund und werden oft ausschließlich in Bezügen auf die Gewaltbereitschaft der Demonstranten verwendet, während in der jeweiligen Textpassage aktivische Sätze mit einem belebten Subjekt dominieren. In Texten aus der russischen Tagespresse hingegen betrifft die Anonymisierung in der Regel längere Textabschnitte, wobei ihre primäre Funktion in der Beibehaltung eines sachlichen und objektivisierten Stils der Berichterstattung besteht.

In vielen Artikeln aus den russischen Zeitungen wird das Ausmaß der Gewaltbereitschaft seitens der ukrainischen Aktivisten mit Hilfe der quantitativen Ikonizität hervorgehoben, indem mehrere Gewalttaten der Protestierenden in direkt aufeinander folgenden Phrasen bzw. Sätzen aufgezählt werden:

Между тем 25 января здание Винниикого областного совета подверглось штурму со стороны украинской оппозиции. В помещении облсовета прозвучали взрывы, распылили газ: в отношении представителей власти было применено насилие. 
[,Inzwischen wurde am 25. Januar das Gebäude des Regionalrats in Winnyzja von der ukrainischen Opposition gestürmt. In den Räumen des Gebäudes kam es zu Explosionen, man versprühte Gas, gegenüber den Vertretern der Behörden wurde Gewalt eingesetzt.']

(Макарычев 2014b, РГ)

Darüber hinaus wird in zahlreichen Texten aus dem russischen Korpus die Brutalität der ukrainischen Aktivisten bereits in Titeln profiliert:

Боевики на Майдане стреляли в стражей порядка спецпатронами

[,Die Kämpfer auf dem Maidan schossen mit Spezialpatronen auf die Ordnungshüter']

(Макарычев 2014a, РГ)

\subsection{Demonstranten als Opfer des Konflikts}

Ein anderer Aspekt des diskursiven Bildes von ukrainischen Aktivisten, der ausschließlich im deutschen und polnischen Korpus präsent ist, steht im Zusammenhang mit der Konzeptualisierung der Protestierenden als Opfer des Konflikts. Zu sprachlichen Mitteln, die das genannte Subbild der Demonstranten konstituieren, gehören Ausdrücke, welche die Handlungen der Gewalttäter profilieren:

In der Ukraine halten Menschen die europäische Fahne hoch, sie werden geschlagen und beschossen.

(Brössler 2014, SZ)

skatowany działacz Majdanu Dmytro Bulatow

[,der gefolterte Maidan-Aktivist Dmitro Bulatow']

(Bielecki 2014, GW)

Der Darstellung der ukrainischen Demonstranten als Opfer des Konflikts dienen auch lexikalische Einheiten, welche die kognitiven Domänen ,Körperverletzung', ,Opfer', und ,Tod' aktivieren:

Erst am Wochenende ist ein weiterer Demonstrant an Schussverletzungen gestorben. Die Zahl der Todesopfer steigt damit auf vier.

(Schuller 2014, FAZ)

Doktor Oteh Musij, koordynujacy działania ekipy medycznej antyrzadowych demonstrantów, poinformowat, że $w$ czwartkowych starciach z milicja zginęto co najmniej 70 uczestników protestów $i$ że ostateczna liczba ofiar może się okazać większa.

[,Der Arzt Oleh Musij, der den medizinischen Dienst der Demonstranten koordiniert hatte, berichtete, dass in den am Donnerstag stattgefundenen Auseinandersetzungen mit der Polizei mindestens 70 Protestierende ums Leben kamen, wobei die endgültige Opferzahl noch höher sein kann.']

(Pomorski 2014, DP)

In manchen Artikeln wird das Ausmaß vom Leiden der Protestierenden mit Hilfe von quantitativer Ikonizität hervorgehoben, indem mehrere Gewalttaten gegenüber den Demonstranten in einem Satz aufgelistet werden:

Immer mehr Menschen werden als vermisst gemeldet, werden geschlagen, festgenommen, unter grotesken Anschuldigungen eingesperrt.

(Sabuschko 2014, FAZ) 
Opozycjoniści odnosili rany i ginęli od kul, polewano ich woda, by zmarli na mrozie, innych torturowano i porzucano w lesie na pewna śmierć.

[,Die Oppositionellen haben Wunden erlitten und fielen Kugeln zum Opfer, sie wurden mit Wasser überschüttet, damit sie im Frost ums Leben kommen, andere wurden gefoltert und im Wald ausgesetzt, wo sie der sichere Tod erwartete.']

(Snyder 2014, GW)

\subsection{Demonstranten als Vertreter von Minderheitsorganisationen, die rechtswidrige Maßnahmen ergreifen}

Ein wichtiger Aspekt des diskursiven Bildes von ukrainischen Aktivisten, der in der russischen Presse besonders deutlich zum Ausdruck kommt, sind Demonstranten als Vertreter von Minderheitsorganisationen, die rechtswidrige Maßnahmen ergreifen. Der Konstruktion des genannten Subbildes dienen zahlreiche Ausdrücke, welche die kognitiven Domänen der Rechtswidrigkeit und der Verletzung der öffentlichen Ordnung aktivieren: „неправительственные организации“ [,illegale Organisationen“] ( $\mathrm{NN}_{7}$ 2014, Известия), „массовые беспорядки“ [,Massenunruhen“] (Макарычев 2014b, РГ), „противоправные действия“ [,rechtswidrige Handlungen'] (Дульман 2014b, РГ).

Darüber hinaus kommen in zahlreichen Artikeln aus Российская Газета und Известия еxplizite Kommentare vor, die auf die Verantwortung der Oppositionsparteien und rechtsradikaler Organisationen für die Veranstaltung der Proteste verweisen:

Государственный переворот на Украине, который попытались совершить при поддержке фашиствующих националистов сторонники нескольких проевропейских партий [...].

[,Der Staatsstreich in der Ukraine, den die Anhänger einiger proeuropäischer Parteien mit Unterstützung der faschistischen Nationalisten anstrebten [...].']

(Шестаков 2014, РГ)

\subsection{Demonstranten als Verräter der eigenen Nation}

Ein weiterer Aspekt des diskursiven Bildes von den ukrainischen Aktivisten, der in der russischen Presse besonders stark hervorgehoben wird, sind Demonstranten als Verräter der eigenen Nation. Der Konstruktion des genannten Subbildes von Protestteilnehmern dienen insbesondere sprachliche Ausdrücke, welche die kognitive Domäne des Verkaufs aktivieren:

Почем продают Украину?

[,Warum verkaufen sie die Ukraine?‘]

(Шестаков 2014, РГ)

$\mathrm{Zu}$ sprachlichen Ausdrucksmitteln, mit deren Hilfe die Protestierenden als Staatsverräter dargestellt werden, gehören auch explizite Kommentare, die auf die Finanzierung der ukrainischen Opposition von westlicher Seite verweisen:

Ранее украинские политики, к примеру, депутат Верховной Рады Олег Царев прямо заявляли, что ведомый Булатовым ,, автомайдан“ и все их акиии финансируются из-за рубежа. 
[,Die ukrainischen Politiker, z. B. der Abgeordnete von Werchowna Rada Oleg Zarew, haben zuvor offen erklärt, dass der von Bulatov organisierte Automaidan und alle seine Aktionen aus dem Ausland finanziert werden.']

(Дульман 2014a, РГ)

Darüber hinaus sind im analysierten Korpus Textstellen zu finden, in denen die Aktivitäten der Demonstranten als Gefahr für die ukrainischen Bürger konzeptualisiert werden:

Радикальные и экстремистские группировки своими действиями несут реальную угрозу жизни миллионам украинских граждан.

[, Radikale und extremistische Gruppierungen bringen durch ihre Aktivitäten das Leben von Millionen ukrainischer Bürger in Gefahr.']

(Алешина/Дульман/Федякина 2014, РГ)

Zur Hervorhebung des Schadenausmaßes in der Ukraine dient die Krankheitsmetapher, die in einigen Artikeln aus dem analysierten Korpus bewusst verwendet wird:

Ведь вся Украина покрыта сетью как чумной оспой, неправительственными организаииями, спонсируемыми Западом.

[,Also ist die ganze Ukraine, wie von Pocken, von Nichtregierungsorganisationen bedeckt, die vom Westen finanziert werden. $\left.{ }^{\circ}\right]$

( $\mathrm{NN}_{7}$, Известия)

\section{$5 \quad$ Schlussfolgerungen}

Aus den oben präsentierten Untersuchungsergebnissen wird ersichtlich, dass zwischen dem im mittel- sowie westeuropäischen und im osteuropäischen Mediendiskurs konstruierten Bild der ukrainischen Demonstranten wesentliche Unterschiede bestehen. Der erste Unterschied betrifft die relative Prägnanz von Zielen der Maidan-Aktivisten. Während in der deutschen und polnischen Tagespresse die Protestierenden als Subjekte konzeptualisiert werden, die bewusst ihre politischen Ziele realisieren, wird dieser Aspekt vom diskursiven Bild der Demonstranten im russischen Subkorpus marginalisiert. Die politischen Postulate der ukrainischen Aktivisten werden in Российская Газета und Известия nur in wenigen Artikeln aus der ersten Phase der Euromaidan-Bewegung thematisiert.

Zweitens werden die Rolle der Protestierenden im Aufbau des ukrainischen Staates und ihre Beziehung zur ukrainischen Bevölkerung im mittel- sowie westeuropäischen und im osteuropäischen Mediendiskurs anders aufgefasst. In der deutschen und polnischen Tagespresse werden die Demonstranten als Vertreter der ukrainischen Gesellschaft dargestellt, was bereits aus zahlreichen metonymischen Bezügen auf die Maidan-Aktivisten ersichtlich wird, in denen die kognitiven Domänen der Menschen und Ukrainer aktiviert werden. In den Vordergrund tritt der Beitrag der Protestierenden zur Aufrechterhaltung der ukrainischen Souveränität sowie der spontane, bürgerliche Charakter der Bewegung. Im russischen Subkorpus werden die Demonstranten hingegen als Vertreter von Minderheitsorganisationen und Verräter der eigenen Nation konzeptualisiert, die eine reale Gefahr für die Ukraine und ihre Bürger darstellen.

Der dritte Unterschied bezieht sich auf die Prägnanz der Gewaltbereitschaft beider Konfliktparteien. Im deutschen und polnischen Mediendiskurs werden die Demonstranten grundsätzlich als Opfer des Konflikts konzeptualisiert. Ihre etwaige Gewaltbereitschaft ist als Reaktion auf die von der Miliz ergriffenen Maßnahmen aufzufassen. In der russischen Tagespresse gehört 
hingegen die Brutalität der Demonstranten zu den wichtigsten Aspekten des Bildes von der ukrainischen Protestbewegung.

Zusammenfassend lässt sich feststellen, dass in den analysierten deutschen und polnischen sowie russischen Tageszeitungen durch die Auswahl sprachlicher Ausdrucksmittel verschiedene Aspekte vom diskursiven Bild der ukrainischen Demonstranten in den Vordergrund gerückt bzw. marginalisiert werden. Dies zeugt von der Tatsache, dass die sprachliche Konstruktion diskursiv zentraler Begriffe im hohen Maße durch kulturspezifische Sichtweisen determiniert ist, die mit den für die unterschiedlichen Kulturräume charakteristischen Weltbildern korrespondieren.

\section{Literaturverzeichnis}

Bartmiński, Jerzy (2006): Językowe podstawy obrazu świata. Lublin: Wydawnictwo UMCS. Bartmiński, Jerzy (2012): „Der Begriff des sprachlichen Weltbildes und die Methoden seiner Operationalisierung“. Text und Diskurs 5: 263-289.

Busse, Dietrich (2008): ,Linguistische Epistemologie. Zur Konvergenz von kognitiver und kulturwissenschaftlicher Semantik am Beispiel von Begriffsgeschichte, Diskursanalyse und Frame-Semantik“. In: Kämper, Heidrun/Eichinger, Ludwig (eds.): Sprache - Kognition Kultur. Sprache zwischen mentaler Struktur und kultureller Prägung. Berlin/New York, de Gruyter: 73-114.

Busse, Dietrich/Teubert, Wolfgang (1994): „Ist Diskurs ein sprachwissenschaftliches Objekt? Zur Methodenfrage der historischen Semantik“. In: Busse, Dietrich/Hermanns, Fritz/Teubert, Wolfgang (eds.): Begriffsgeschichte und Diskursgeschichte. Methodenfragen und Forschungsergebnisse der historischen Semantik. Opladen, Westdeutscher Verlag: 10-28.

Czachur, Waldemar (2011): Diskursive Weltbilder im Kontrast. Linguistische Konzeption und Methode der kontrastiven Diskursanalyse deutscher und polnischer Medien. Wrocław: ATUT.

Czachur, Waldemar (2013): „Das diskursive Weltbild und seine kognitionstheoretische Fundierung in der Diskurslinguistik“. Studia Germanica Gedanensia 29: 186-197.

Foucault, Michel (1991): Die Ordnung des Diskurses. Frankfurt: Fischer.

Fraas, Claudia (2005): „Schlüsselkonzepte als Zugang zum kollektiven Gedächtnis. Ein diskurs- und frameanalytisch basierter Ansatz“. Deutsche Sprache 33/3: 242-257.

Fraas, Claudia/Meier, Stefan (2004): „Diskursive Konstruktion kollektiven Wissens on- und offline“. OBST. Osnabrücker Beiträge zur Sprachtheorie 68: 77-102.

Gaweł, Agnieszka (2016): „Zur Anwendbarkeit der Kognitiven Linguistik in der Diskursanalyse. Bisherige Erkenntnisse der polnischen Germanistik und Perspektiven“. In: Duś, Magdalena/Kołodziej, Robert/Rojek, Tomasz (eds.): Wort-Text-Diskurs. Frankfurt a. M. etc., Lang: 351-364.

Gaweł, Agnieszka (2020): „Zur Rekonstruktion der kognitiven Definition des Briten - anhand des Brexit-Diskurses der Tageszeitung“ (im Druck).

Heinemann, Margot/Heinemann, Wolfgang (2002): Grundlagen der Textlinguistik. Interaktion - Text-Diskurs. Tübingen: Niemeyer. 
Humboldt, Wilhelm von (1836): Über die Verschiedenheit des menschlichen Sprachbaues und ihren Einfluß auf die geistige Entwicklung des Menschengeschlechts. Berlin: Dümmler. opacplus.bsb-muenchen.de/title/BV001604808 [29.09.2020].

Langacker, Ronald W. (2001): „Discourse in Cognitive Grammar“. Cognitive Linguistics 122/2001: 143-188.

Langacker, Ronald W. (2008): Cognitive Grammar. A Basic Introduction. Oxford: Oxford University Press.

Pędzisz, Joanna (2012): „Diskursthema und Themen im Diskurs. Zur thematischen Profilierung der diskursiven Wirklichkeit“". Text und Diskurs 5: 231-247.

Pędzisz, Joanna (2014): „Diskurs und discourse: Versuch einer Integration der Diskursanalyse und Cognitive Grammar“. Zeitschrift des Verbandes Polnischer Germanisten 3/2014, 3: 249-260.

Spieß, Constanze (2011): Diskurshandlungen. Theorie und Methode linguistischer Diskursanalyse am Beispiel der Bioethikdebatte. Berlin/New York: de Gruyter.

Tokarski, Ryszard/Nowak, Paweł (1999): „Językowy obraz świata a perswazyjna funkcja tekstów (o poszukiwaniu wspólnoty kulturowej)". In: Greszczuk, Barbara (ed.): Język Teoria - Dydaktyka. Materiały 21. konferencji językoznawczej zorganizowanej w Trzcinicy k. Jasła w dniach 27-29 maja 1998 roku. Rzeszów, Wydawnictwo Wyższej Szkoły Pedagogicznej: 207-215.

Warnke, Ingo (2002): „Adieu Text - bienvenue Diskurs?“ In: Fix, Ulla et al. (eds.): Brauchen wir einen neuen Textbegriff? Antworten auf eine Preisfrage. Frankfurt a. M., Lang: 1 25-141.

Weisgerber, Leo (1950): Die Muttersprache im Aufbau unserer Kultur. Düsseldorf: Schwann. Whorf, Benjamin Lee (1956): Language, Thought, and Reality: Selected Writings of Benjamin Lee Whorf, (ed.) von John B. Carroll. Cambridge, MA: MIT Press.

\section{Quellenverzeichnis}

Алешина, Марина/Дульман, Павел/Федякина, Анна (2014): «Украинская ночь: конец тишины». РГ 20.02.2014. rg.ru/2014/02/19/ukraina-site.html [02.08.2019].

Bielecki, Tomasz (2014): „Ważna deklaracja Van Rompuya: - Przyszłość Ukrainy należy do Unii Europejskiej”. GW 01.02.2014. wyborcza.pl/1,75477,15383812,Wazna_deklaracja_ Van_Rompuya_Przyszlosc_Ukrainy.html [02.08.2019].

Bielecki, Tomasz/Tymots, Ihor (2014): „Ukraina trzeszczy w szwach. Bunt na zachodzie“. GW 24.01.2014. wyborcza.p1/1,75399,15334408,Ukraina_trzeszczy_w_szwach_Bunt_na_ zachodzie.html [05.10.2020].

Brössler, Daniel (2014): „Warum es in der Ukraine um Europas Zukunft geht“. SZ 23.01.2014. sueddeutsche.de/politik/krise-in-kiew-warum-es-in-der-ukraine-um-europas-zukunft-geht1.1870629 [13.02.2019].

Дульман, Павел (2014а): «МВД Украины: Беспорядки в Киеве планируются заранее». РГ 31.01.2014. rg.ru/2014/01/31/kiev1-site-anons.html [02.08.2019].

Дульман, Павел (2014b): «Янукович освободил участников протестов». РГ 31.01.2014. rg.ru/2014/01/31/kiev-site-anons.html [02.08.2019].

Дульман, Павел/Макарычев, Максим (2014): «Хроника беспорядков в Киеве». РГ 18.02.2014. rg.ru/2014/02/18/hronika-site-anons.html [01.04.2019]. 
Junko, Jarosław (2014a): „Cztery osoby w stanie ciężkim po zamieszkach w Kijowie”. DP 20.01.2014. dziennikpolski24.pl/pl/aktualnosci/na-biezaco/1301588-ukraina-cztery-osobyw-stanie-ciezkim-po-zamieszkach-w-kijowie.html [02.08.2019].

Junko, Jarosław (2014b): „Ludzie zajmują budynki państwowe we Lwowie i Równem”. DP 23.01.2014. dziennikpolski24.pl/pl/aktualnosci/na-biezaco/1301980-ukraina-ludziezajmuja-budynki-panstwowe-we-lwowie-i-rownem.html [02.08.2019].

Kahlweit, Cathrin (2014a): „Ein unmoralisches Angebot.” SZ 26.01.2014. sueddeutsche.de/politik/machtkampf-in-der-ukraine-ein-unmoralisches-angebot-1.1871955 [02.08.2019].

Kahlweit, Cathrin (2014b): „Wie in Kiew die Fronten verwischen”. SZ 20.02.2014. sueddeutsche.de/politik/waffenruhe-in-ukraine-gebrochen-der-maidan-ist-wieder-kampfzone1.1893844 [11.03.2019].

Knap, Włodzimierz (2014): „Ukraina przeżywa ciężki czas i czeka na przełom”. DP 27.01.2014. dziennikpolski24.pl/pl/aktualnosci/swiat/1302321-ukraina-przezywa-ciezkiczas-i-czeka-na-przelom.html [02.08.2019].

Kołodyński, Michał (2013): „Protestuje kilkaset tysięcy osób”. DP 05.12.2013. dziennikpolski24.pl/protestuje-kilkaset-tysiecy-osob/ar/3291662\#aktualnosci [08.04.2019].

Kublik, Andrzej (2014): „Pożyczki, ceny gazu i szantaż. Jak Moskwa manipuluje Ukrainą”. GW 20.02.2014. wyborcza.pl/1,75399,15491853,Pozyczki_ceny_gazu_i_szantaz_ Jak_Moskwa_manipuluje.html [25.01.2019].

Лимонов, Эдуард (2014): »Войнуха, войнища, война». Известия 19.02.2014. iz.ru/news/566146 [03.04.2019].

Лубенский, Андрей/Соколовская, Янина (2013): «Киевский майдан ждет вестей из Москвы». Известия 17.12.2013. iz.ru/news/562685 [02.08.2019].

Макарычев, Максим (2013): «Майданофобия». РГ 27.11.2013. rg.ru/2013/11/27/kievsite.html [25.01.2019].

Макарычев, Максим (2014a): «Боевики на Майдане стреляли в стражей порядка спецпатронами». РГ 20.02.2014. rg.ru/2014/02/20/patroni-site-anons.html [01.04.2019].

Макарычев, Максим (2014b): «Украина: хроника воскресных событий». РГ 26.01.2014. rg.ru/2014/01/26/ukraina-site.html [02.08.2019].

$\mathrm{NN}_{1}$ (2014): „Blutbad in Kiew”. SZ 20.02.2014. sueddeutsche.de/politik/demonstrationenblutbad-in-kiew-dpa.urn-newsml-dpa-com-20090101-140219-99-09751 [02.08.2019].

$\mathrm{NN}_{2}$ (2014): „Die Ungeduld der Meute“. FAZ 24.01.2014. faz.net/aktuell/politik/proteste-inkiew-die-ungeduld-der-meute-12766988.html [11.03.2019].

$\mathrm{NN}_{3}$ (2014): „EU droht Janukowitsch”. FAZ 22.01.2014. faz.net/aktuell/politik/tote-bei-protesten-in-ukraine-eu-droht-janukowitsch-12763427.html [02.08.2019].

$\mathrm{NN}_{4}$ (2014): „Straßenschlachten in Kiew“. SZ 20.01.2014. sueddeutsche.de/politik/unruhenin-der-ukraine-strassenschlachten-in-kiew-1.1867692 [02.08.2019].

$\mathrm{NN}_{5}$ (2013): „Ukrainische Polizei geht gewaltsam gegen Demonstranten vor“. SZ 24.11.2013. sueddeutsche.de/politik/ukraine-zehntausende-protestieren-gegen-abkehr-von-der-eu1.1826224 [19.04.2019].

$\mathrm{NN}_{6}$ (2014): «Активисты майдана захватили в плен 10 военнослужащих». Известия 20.02.2014. iz.ru/news/566239 [03.04.2019].

$\mathrm{NN}_{7}$ (2014): «Александр Проханов: «Майдан - не стихия, а организация»». Известия 28.01.2014. iz.ru/news/564729 [05.10.2020]. 
$\mathrm{NN}_{8}$ (2013): «Митингующие в центре Киева разбили окна городской администрации». РГ 01.12.2013. rg.ru/2013/12/01/miting-anons.html [28.09.2020].

Petrowskaja, Katja (2014): „Die Angst, der Stolz, der Zorn“. FAZ 09.02.2014. faz.net/aktuell/feuilleton/buecher/themen/proteste-in-kiew-die-angst-der-stolz-der-zorn-12791661.html [08.04.2019].

Pomorski, Michał (2014): „Krwawa masakra na kijowskim Majdanie”. DP 21.02.2014. dziennikpolski24.pl/krwawa-masakra-na-kijowskim-majdanie/ar/3340381\#aktualnosci [19.04.2019].

Radziwinowicz, Wacław (2014): „Wacław Radziwinowicz pisze z Majdanu: Strzelali w moją stronę i korespondenta TVN". GW 20.02.2014. wyborcza.pl/1,75399,15493125,Waclaw_ Radziwinowicz_pisze_z_Majdanu_Strzelali_w.html [15.03.2019].

Sabuschko, Oksana (2014): „Dieses Drehbuch schrieb ein Irrer”. FAZ 29.01.2014. faz.net/aktuell/feuilleton/ukraine-dieses-drehbuch-schrieb-ein-irrer-12775449.html [02.08.2019].

Schuller, Konrad (2014): „Schlacht um das ukrainische Haus“. FAZ 26.01.2014. faz.net/aktuell/politik/ausland/proteste-in-kiew-schlacht-um-das-ukrainische-haus-12770095.html [02.08.2019].

Snyder, Timothy (2014): „Majdan? Zniszczyć!” GW 21.02.2014. wyborcza.pl/magazyn/1,124059,15505912,Majdan_Zniszczyc_html [08.04.2019].

Соколовская, Янина (2014): «Толпа в Киеве забила насмерть компьютерщика из Партии регионов». Известия 18.02.2014. iz.ru/news/566112 [03.04.2019].

Шестаков, Евгений (2014): «Почем продают Украину?» РГ 03.02.2014. rg.ru/2014/02/02/ykraina-site.html [02.08.2019].

Вдовин, Владислав (2013): «Город победившего майдана». Известия 08.12.2013. $\mathrm{iz.ru} / \mathrm{news} / 562112$ [27.01.2019].

Wieliński, Bartosz T. (2014): „Dziennikarz z Kijowa specjalnie dla 'Wyborczej’: Ukraińców nie da się złamać”. GW 30.01.2014. wyborcza.pl/1,75477,15358151, Dziennikarz_z_Kijowa_specjalnie_dla_Wyborczej__Ukraincow.html [02.08.2019]. 